\title{
Prognosis of Patients With Cardiogenic Shock Following Acute Myocardial Infarction: The Difference Between ST-Segment Elevation Myocardial Infarction And Non-ST-Segment Elevation Myocardial Infarction
}

Ming-Lung Tsai

New Taipei Municipal TuCheng Hospital

Ming-Jer Hsieh

Chang Gung Memorial Hospital, Chang Gung University College of Medicine

Chun-Chi Chen

Chang Gung Memorial Hospital, Chang Gung University College of Medicine

Victor Chien-Chia Wu

Chang Gung Memorial Hospital, Chang Gung University College of Medicine

Wen-Ching Lan

Chang Gung Memorial Hospital

Yu -Tung Huang

Chang Gung Memorial Hospital

I-Chang Hsieh

Chang Gung Memorial Hospital, Chang Gung University College of Medicine

Shang-Hung Chang ( $\sim$ afen.chang@gmail.com )

Chang Gung Memorial Hospital, Chang Gung University College of Medicine

\section{Research Article}

Keywords: Acute myocardial infarction, Cardiogenic shock, Critical care

Posted Date: September 13th, 2021

DOI: https://doi.org/10.21203/rs.3.rs-841985/v1

License: (c) (i) This work is licensed under a Creative Commons Attribution 4.0 International License.

Read Full License 
Title: Prognosis of Patients with Cardiogenic Shock Following Acute Myocardial Infarction: The Difference Between ST-Segment Elevation Myocardial Infarction and Non-ST-Segment Elevation Myocardial Infarction

Authors: Ming-Lung Tsai ${ }^{1,2}$, Ming-Jer Hsieh ${ }^{2}$, Chun-Chi Chen ${ }^{2}$, Victor Chien-Chia $\mathrm{Wu}^{2}$, Wen-Ching Lan ${ }^{3}$, Yu-Tung Huang ${ }^{3}$, I-Chang Hsieh ${ }^{2}$, Shang-Hung Chang ${ }^{2,3, *}$

\section{Affiliation:}

${ }^{1}$ Division of Cardiology, New Taipei Municipal TuCheng Hospital, Taiwan

${ }^{2}$ Division of Cardiology, Department of Medicine, Chang Gung Memorial Hospital, and Chang Gung University College of Medicine, Taoyuan, Taiwan

${ }^{3}$ Center for Big Data Analytics and Statistics, Chang Gung Memorial Hospital, Linkou Medical Center, Taoyuan, Taiwan

\section{Contributions}

CCC, ICH, MLT, and SHC conceived and designed the study. MLT, MJH, and CCW, drafted the manuscript. WCL and YTH collected, analyzed, interpreted the data, and performed the statistical analyses. All authors reviewed the manuscript.

*Correspondence author: Shang-Hung Chang

Tel: $+886-3-3289134$

E-mail address: afen.chang@gmail.com 
Postal address: No.5, Fu-Hsing Street, Kwei-Shan, Taoyuan, Taiwan

Keywords: Acute myocardial infarction, Cardiogenic shock, Critical care 


\section{Acknowledgment}

The authors thank the statistical assistance and wish to acknowledge the support of the Maintenance Project of the Center for Big Data Analytics and Statistics (Grant CLRPG3D0046) at Chang Gung Memorial Hospital for study design and monitor, data analysis and interpretation.

\section{Availability of data and materials}

The datasets used and/or analyzed during the present study are available from the corresponding author on reasonable request.

\section{Funding}

This work was supported by the grant from Chang Gung Memorial Hospital [grant number: CORPG3G0291,

CORPG3C0162, CMRPG3I0092].

\section{Ethics declarations}

\section{Ethics approval and consent to participate}

This study was approved by the Chang Gung Memorial Hospital Institutional Review Board (201700965B0C502) and waivered of informed consent.

\section{Consent for publication}

Not applicable.

\section{Competing interests}

The authors declare that they have no competing of interests. 


\section{Abstract}

Background: Acute myocardial infarction (AMI) complicated with cardiogenic shock has high mortality and is a challenging topic even in the revascularization era. We conducted this study to understand patients' outcomes. Method: We retrospectively analyzed electronic medical records data from 1,175 patients with AMI complicated with cardiogenic shock developed within 3 days of admission to a multicenter medical care system between January 1, 2000, and July 31, 2018. AMI patients were classified into ST-segment elevation myocardial infarction (STEMI) or non-ST-segment elevation myocardial infarction (NSTEMI) group. The short-term and 1-year mortality and adverse event after the index admission were analyzed via logistic regression and Cox proportional hazards model. Results: Comparing to NSTEMI, patients with STEMI tended to be younger $(65.68 \pm 14.05$ vs. $70.70 \pm 12.99, p<.001)$, men $(73.29$ vs. $60.87, p<.001)$, and have fewer underlying chronic diseases. Short-term mortality at index hospitalization was $14.83 \%$ in the STEMI group and $21.30 \%$ in the NSTEMI group; long-term mortality was $17.06 \%$ for the STEMI group and $24.13 \%$ for the NSTEMI group. No difference was observed between the 2 groups for patients who developed a cerebral vascular accident (CVA) during the admission period; however, the major bleeding rate and gastrointestinal bleeding rate were higher in the STEMI group (2.66 vs. $0.22, p=.014 ; 3.36$ vs. $0.22, p$ $=.007$, respectively). Conclusion: In patients with AMI with cardiogenic shock, NSTEMI was associated with a significantly higher mortality rate in both the short- and long-term results. Age and respiratory failure were the most significant risk factors for short-term mortality. Revascularization may be beneficial for the short-term outcome but did not reach significance in multivariable analysis. 


\section{Introduction}

Cardiogenic shock after acute myocardial infarction (AMI) was fatal before revascularization treatment was implemented as a routine procedure, with mortality rates higher than $50 \%$ without revascularization. ${ }^{1}$ With the development of prevention strategies and the adoption of revascularization, the survival rates of patients improved; however, AMI with cardiogenic shock remains a challenge situation despite improvements in treatment modalities and supporting systems. ${ }^{2}$

Outcomes for patients with AMI vary according to the clinical setting. Patients with ST-segment elevation myocardial infarction (STEMI) and non-STEMI (NSTEMI) have different characteristics and disease specificity, which could explain their different short-term and long-term survival and prognoses. A study using the OPERA registry compared the 1-year prognosis of patients with STEMI or NSTEMI and demonstrated a similar but numerically higher STEMI in-hospital mortality rate (4.6\% vs. $4.3 \%)$ and a marginally lower 1 -year death rate $(9.0 \%$ vs. $11.6 \%, \log$-rank $p=.009) .{ }^{3}$

Among the survivors of AMI complicated by cardiogenic shock who are discharged from the hospital, approximately $20 \%$ are readmitted within 30 days, mainly because of heart failure (HF) and newly

developed AMI. ${ }^{4}$ Long-term outcome studies for these patients are limited, especially for patients in East Asia. Furthermore, the prognosis between STEMI and NSTEMI with cardiogenic shock is unclear. To address this issue, we conducted this study to understand the prognoses, patient characteristics, and risk factors among patients hospitalized for AMI complicated with cardiogenic shock.

\section{Methods}

\section{Data Source}

This study was conducted using the Chang-Gung Research Database (CGRD). CGRD is a de-identified database derived from the original electronic medical records of Chang-Gung Memorial Hospital system (CGMHs), which comprises 7 medical institutes located from the northeastern to southern regions of Taiwan. A large range of medical services is performed in CGMHs; clinical and scientific studies based on CGRD are of high quality and have been validated. ${ }^{5}$ The Chang Gung Memorial Hospital Institutional Review 
board approved this study and waiver of informed consent was granted, 201700965B0C502. The

information and records of patients were anonymized and de-identified prior to analysis. The authors are responsible for the design and conduct, drafting, and editing of this manuscript and its contents.

\section{Study Group and Cohort Definition}

From CGRD, we identified patients who were admitted for AMI between January 1, 2000, and July 31, 2018. Patients who hospitalization via our emergency department with AMI complicated with documented cardiogenic shock events required inotropic agent, intra-aortic balloon pump (IABP), or extracorporeal membrane oxygenation (ECMO) in the first 3 days of index date were enrolled into the study.

The index hospitalization was defined as the date when the patient was admitted for AMI. The follow-up period was defined as the date of the index hospitalization to the date of death or loss of follow-up or until July 31, 2018, whichever occurred first. AMI patients were classified into STEMI or NSTEMI group according to the International Classification of Diseases (ICD), $9^{\text {th }}$ and $10^{\text {th }}$ Revision codes. STEMI was defined based on ICD-9 codes of 410.0x, 410.1x-410.6x, and 410.8x or ICD-10 codes of I21.0*-I21.3*, I22.0, I22.1, I22.8, and I22.9. NSTEMI was defined based on the ICD-9 code of 410.7x or the ICD-10 codes of I21.4* and I22.2. Cardiogenic shock was defined as (1) the use of inotropic agents, including dopamine, dobutamine, norepinephrine, or epinephrine, or (2) the implementation of an IABP or ECMO to stabilize hemodynamics. Patients were excluded if they were lost to follow-up 90 days after discharge.

\section{Outcomes and Covariate Measurements}

The primary outcomes were 30-day mortality or mortality at index hospitalization. Secondary outcomes included new-onset hemodialysis, new-onset stroke, major bleeding, gastrointestinal (GI) bleeding, pneumonia, or sepsis within 30 days after the index admission or during the index hospitalization period. We also analyzed the 1-year outcomes including recurrent myocardial infarction, coronary revascularization, death, stroke, or bleeding events. Coronary revascularization was defined as percutaneous coronary revascularization or coronary artery bypass surgery $(\mathrm{CABG})$. Stroke included ischemic, hemorrhagic, or unspecified stroke. Death was identified according to the registry data of CGRD, including death or critical discharge. Secondary outcomes, such as HF admission and major bleeding, were specifically evaluated. 
Major bleeding was defined as life threatening or critical bleeding requiring blood transfusion 2 or more unit. All these outcomes and covariate's definition were also defined by ICD-9 and ICD-10 codes. The detailed coding was list in supplement.

Subgroup analysis for short-term prognosis of STEMI or NSTEMI were also conducted. Age, gender, IABP, ECMO, revascularization during the index admission, and comorbidity were listed for predictor. Other covariate included chronic diseases, such as diabetes mellitus, hypertension, hyperlipidemia, atrial fibrillation, chronic obstructive pulmonary disease (COPD), chronic kidney disease (CKD), peripheral artery occlusive disease (PAOD), gout, and malignancy. In addition, patients the history of cardiovascular diseases, such as HF, myocardial infarction (MI), cerebral vascular accident (CVA), and previous treatment, such as percutaneous coronary intervention (PCI) and coronary artery bypass grafting (CABG) were also defined as covariates.

\section{Statistical Analysis}

We compared the clinical characteristics between the study groups using $\chi^{2}$ test for categorical variables, independent sample $t$ test for continuous variables, and Kruskal-Wallis test for the median values. Kaplan-Meier analysis was conducted to estimate the cumulative mortality rate. For compared the primary and secondary outcomes for 30-day or in-hospital death between STEMI and NSTEMI, logistic regression was employed for these analyses. In a further subgroup analysis, we developed a multivariate Cox proportional hazards model to identify the factors associated with overall mortality for patients with cardiogenic shock of STEMI or NSTEMI. The results are presented as adjusted odds ratio (OR) for logistic regression or adjusted hazard ratio (HR) for Cox model with corresponding 95\% confidence intervals (CIs).

\section{Results}

A total of 1175 patients with AMI complicated with cardiogenic shock were enrolled into our study, with 715 patients with STEMI and 460 patients with NSTEMI. In our study population, comparing to NSTEMI, patients with STEMI complicated with cardiogenic shock tended to be younger $(65.68 \pm 14.05$ vs. $70.70 \pm 12.99, p<.001)$, men (73.29 vs. $60.87, p<.001)$, and have fewer underlying chronic diseases, such 
as diabetes mellitus, hypertension, hyperlipidemia, atrial fibrillation, COPD, CKD, PAOD, gout, and malignancy (Table 1). Moreover, patients with STEMI were less likely to have a history of cardiovascular diseases, such as HF, MI, CVA, or PCI. Primary outcomes were defined as 30-day mortality or mortality at index hospitalization (Table 2). Mortality at index hospitalization was $14.83 \%$ for the STEMI group and $21.09 \%$ for the NSTEMI group; 30-day mortality was $14.27 \%$ for the STEMI group and $19.13 \%$ for the NSTEMI group. Moreover, we evaluated secondary outcomes as events at index hospitalization, including new hemodialysis, new-onset CVA, major bleeding, GI bleeding, pneumonia, and sepsis. The new-onset dialysis rate during index hospitalization was higher in the NSTEMI group; moreover, pneumonia and sepsis were higher in the NSTEMI group. No difference was observed between the 2 groups for patients with CVA during the admission period; however, the major bleeding rate and GI bleeding rate were higher in the STEMI group (2.66 vs. $0.22, p=.014 ; 3.36$ vs. $0.22, p=.007$; respectively).

One-year follow-up events for patients discharged from index hospitalization are displayed in Table 3. For these patients, the prevalence of recurrent NSTEMI episodes was lower in the STEMI group (1.64 vs. $4.41, p=.013)$. Readmission for HF (9.03 vs. $12.95, p=.057)$ and all-cause mortality $(2.63$ vs. $4.13, p=.202)$ showed better outcomes in the STEMI group. However, the requirement of percutaneous revascularization was higher in the STEMI group (9.52 vs. 5.51, $p=.027)$. The overall 1-year cumulative mortality rate for patients with STEMI and NSTEMI was $17.06 \%$ and 24.13\%, respectively, as shown in Figure 1.

Subgroup analysis for predicting the risk of short-term mortality for STEMI or NSTEMI are shown in Table $4 \mathrm{a}$ and $4 \mathrm{~b}$, respectively. Univariate analysis showed that in the STEMI group, age, requirement of ECMO, and intubation significantly increased mortality risk; male gender and revascularization were suggested to be protective factors. Age, ECMO, intubation, and gender showed predictive power in multivariable analysis of the STEMI group; revascularization showed a potential benefit but did not reach significance. For the NSTEMI group, age and intubation were significant risk factors for short-term mortality; revascularization could be beneficial but did not reach significance in multivariate analysis. Subgroup analysis results for patients with different age groups are listed in Figure 2a and Figure 2 b. Compared with younger patients, those older than 55 years had a significantly higher mortality rate in both 
the STEMI and NSTEMI groups.

\section{Discussion}

In this study, 30-day or index hospitalization mortality remained high in patients with Killip IV AMI, especially in patients with NSTEMI, with a mortality rate of $21.30 \%$. The outcome difference between STEMI and NSTEMI varied across studies. Upon comparing patients with STEMI and NSTEMI with cardiogenic shock, we found that 30-day or index hospitalization mortality of the STEMI group was significantly lower than that of the NSTEMI group (14.83\% vs. $21.30 \%, p=.004)$. Moreover, 1 -year follow-up demonstrated worse overall survival outcomes for NSTEMI. Prognosis of AMI under different setting varied in previous studies. The OPERA registry from France includes data from 2176 patients with STEMI or NSTEMI, and the study based on the registry demonstrated a higher in-hospital mortality $(4.6 \%$ vs. $4.3 \%)$ but a lower 1 -year death rate $(9.0 \%$ vs. $11.6 \%)$ for STEMI. ${ }^{3}$ In addition, a nationwide registry study in Korea showed that among patients with AMI, a higher 30-day cardiac death rate (8.5\% vs. 5.7\%, respectively) and 1-year cardiac mortality (12.3\% vs. 9.5\%, respectively) were seen in the STEMI group than in the NSTEMI group. ${ }^{6}$ Furthermore, a report from Beijing demonstrated higher short-term mortality in the STEMI group than in the NSTEMI group (6.6\% vs. $5.3 \%$, respectively). ${ }^{7}$ However, these trials did not focus on patients with AMI with cardiogenic shock.

Patients with NSTEMI and STEMI have different characteristics; moreover, their outcomes vary in different settings. The higher mortality rate of patients with NSTEMI could be due to the presence of more comorbidities. In our analysis, patients in the NSTEMI group tended to be older than those in the STEMI group. Patients with NSTEMI had significantly more number of comorbidities, including diabetes, hypertension, dyslipidemia, COPD, CKD, PAOD, and gout. Moreover, previous cardiovascular disease events were higher among patients with NSTEMI. The baseline differences between the STEMI and NSTEMI groups could influence the short-term outcome and 1-year result. In our study, under the setting of cardiogenic shock following AMI, the prognosis was worse for the NSTEMI group in both short-term and long-term follow-ups. We found that patients with AMI with cardiogenic shock could not survive the 
NSTEMI and have increased mortality rate because of underlying conditions.

Moreover, patients with cardiogenic shock in the NSTEMI group had higher risks of pneumonia, sepsis, and new-onset hemodialysis due to age and underlying comorbidities. Furthermore, for the 1-year outcome analysis, patients with NSTEMI had a significantly higher rate of recurrent NSTEMI events and a marginally significant HF readmission rate. Our result is similar to the results of analysis of patients with AMI complicated with cardiogenic shock from the Unites States Nationwide Readmissions Database, which showed a lower 30-day admission rate in the STEMI group (OR: 0.91, CI: 0.82-1.00, $p=.042) .{ }^{4}$ These results suggest that patients in the NSTEMI group require more attention for long-term HF and care for ischemic heart disease.

In our analysis, patients of AMI complicated with cardiogenic shock had significant mortality risks during the first 30 days, which is similar to the IABP-Shock II trial. Studies have shown that the first month after AMI was the most critical period for the patients, and the mortality curve could be smoothened after that ${ }^{8}$. These suggested that the initial treatment and care for these patients are important. Devices or inotropic agent support for these patients are used as life-saving implementations or measures, and ECMO assisted cardiogenic shock showed promising short-term outcomes. ${ }^{9}$ Previous report revealed the use of ECMO were usually limited in the first two weeks, especially in the mortality ones. ${ }^{10}$ These could also explain why the highest mortality risk occurred within the first 30 days.

Interestingly, the number of short-term bleeding, including major and GI bleeding, events was significantly higher in the STEMI group. The higher number of bleeding events in the STEMI group than in the NSTEMI group has been shown in a previous study comparing the risk of bleeding in acute coronary syndrome (ACS). ${ }^{11}$ Patients with more comorbidities were thought to have higher bleeding risk; however, their bleeding rate was lower, although the NSTEMI group had more comorbidities and older age. This result may be partially explained by the use of different procedures or medications between the groups. In both groups, patients had similar rates of PCI and CABG; however, patients in the STEMI group had a higher rate of IABP $(24.48 \%$ vs. $21.52 \%, p=.2425)$ or ECMO $(6.85 \%$ vs. $4.13 \%, p=.0511)$. The use of the 
intravascular supporting system is associated with longer coverage and higher serum levels of antithrombotic agents. Patients in the STEMI group had a higher intravascular supporting system-usage rate and potential higher potency antiplatelet or anticoagulation usage, which could result in more bleeding events.

Subgroup analysis showed the potential benefit of revascularization for patients with AMI complicated with cardiogenic shock. PCI has been reported to be associated with lower mortality in AMI patients complicated with cardiogenic shock, ${ }^{12,13}$ even in older patients who received percutaneous revascularization, with the benefit of decreasing the mortality rate by up to $50 \% .{ }^{14}$ Although our report showed the benefit for both groups (STEMI, HR: 0.35, 95\% CI: 0.23-0.54, $p<.0001$; NSTEMI, HR: 0.37, 95\%: CI 0.24-0.57, $p$ $=.0001)$, the findings did not reach significance in multivariate analysis.

Furthermore, we found that age was a significant predictor of worse prognosis. The relationship of age with short-term outcomes was further analyzed. The risk significantly increased with age. When comparing patients older than 85 years with those younger than 55 years, the short-term risk increased to 3.42 times (HR 3.42, 1.69-6.94. $p=.001)$ in the STEMI group and 7.32 times $($ HR 7.32, 2.51-21.34, $p<.001)$ in the NSTEMI group. Age remained an important risk factor for mortality among patients with AMI complicated with shock. For older patients, this critical condition could lead to a poor prognosis. In addition to age, respiratory failure requiring intubation was a significant predictor of short-term mortality in both the STEMI and NSTEMI groups. The ECMO usage rate was higher in the STEMI group; however, the use of ECMO was associated with higher mortality in this group. Despite the use of inotropic agents or other support, patients with ECMO may have worse outcomes because ECMO is reserved for patients requiring resuscitation.

Finally, among patients with STEMI-related shock, male gender could be a protective factor for short-term outcomes. This gender difference-related outcome has been observed in previous studies of ACS with cardiogenic shock. ${ }^{15,16}$ Other studies focusing on STEMI with gender difference have also shown this phenomenon. ${ }^{17,18}$ The possible causes include older age and more comorbidities in female patients with 
ACS. However, a further study on STEMI in younger patients showed worse short-term outcomes in female patients after adjustment for demographics, comorbidities, medications, and severity of coronary disease. ${ }^{19}$ Furthermore, in the IABP-SHOCK II trial, no gender-related difference was seen in the clinical outcomes after multivariable adjustment for baseline characteristics. ${ }^{15}$ However, the IABP-SHOCK II trial, including both STEMI and NSTEMI cases, and gender-associated outcome differences were less significant for NSTEMI in our study. Therefore, further studies are required to evaluate this phenomenon.

\section{Limitations}

In this database analysis study, we evaluated patients with AMI with cardiogenic shock. The retrospective design limited our ability to enroll patients randomly therefore the selection bias. The claim-based database did not include personal information such as tobacco use, lifestyle, and family history of cardiovascular disease. Information on the daily physical activity was also unavailable in our database. Besides, the diagnoses of comorbidities were based on ICD-9 or ICD-10 codes and it was difficult to distinguish underlying disease severity. Finally, we did not analyze detailed medication usage among different groups of patients, including different oral antihyperglycemic agents, antiplatelets, or anticoagulants, which could be associated with the differences in outcomes.

\section{Conclusion}

We demonstrated the outcomes of STEMI and NSTEMI complicated with shock. The results revealed that mortality remained high in patients with AMI complicated with cardiogenic shock. Moreover, compared with STEMI, NSTEMI was associated with a significantly higher mortality rate in both short-term and long-term results. Furthermore, age and respiratory failure were the most significant risk factors for short-term mortality. Female gender was also a predictor of worse prognosis for STEMI with cardiogenic shock. Finally, revascularization showed a potential benefit for short-term outcomes but did not reach significance in multivariable analysis. 

Table 1. Baseline clinical characteristics of the patients.

Table 2. Outcomes for 30-day or index hospitalization mortality.

Table 3. Subgroup analysis for 1-year follow-up cases.

Table 4a. Subgroup analysis of predictors of 30-day or in-hospital mortality in ST-elevation myocardial infarction (STEMI).

Table 4b. Subgroup analysis of predictors of 30-day or in-hospital mortality in non-ST-elevation myocardial infarction (NSTEMI).

Figure 1. 1-year cumulative mortality of STEMI and NSTEMI patients complicated with cardiogenic shock

Figure 2a. Subgroup analysis of short-term mortality for different age groups in STEMI.

Figure 2b. Subgroup analysis of short-term mortality for different age groups in NSTEMI. 


\section{Reference}

1. Holmes DR, Jr., Bates ER, Kleiman NS, Sadowski Z, Horgan JH, Morris DC, Califf RM, Berger PB, Topol EJ. Contemporary reperfusion therapy for cardiogenic shock: the GUSTO-I trial experience. The GUSTO-I Investigators. Global Utilization of Streptokinase and Tissue Plasminogen Activator for Occluded Coronary Arteries. J Am Coll Cardiol 1995;26:668-674.

2. Chung SY, Tong MS, Sheu JJ, Lee FY, Sung PH, Chen CJ, Yang CH, Wu CJ, Yip HK. Short-term and long-term prognostic outcomes of patients with ST-segment elevation myocardial infarction complicated by profound cardiogenic shock undergoing early extracorporeal membrane oxygenator-assisted primary percutaneous coronary intervention. Int J Cardiol 2016;223:412-417.

3. Montalescot G, Dallongeville J, Van Belle E, Rouanet S, Baulac C, Degrandsart A, Vicaut E, Investigators $O$. STEMI and NSTEMI: are they so different? 1 year outcomes in acute myocardial infarction as defined by the ESC/ACC definition (the OPERA registry). Eur Heart J 2007;28:1409-1417.

4. Mahmoud AN, Elgendy IY, Mojadidi MK, Wayangankar SA, Bavry AA, Anderson RD, Jneid H, Pepine CJ. Prevalence, Causes, and Predictors of 30-Day Readmissions Following Hospitalization With Acute Myocardial Infarction Complicated By Cardiogenic Shock: Findings From the 2013-2014 National Readmissions Database. J Am Heart Assoc 2018;7.

5. Tsai MS, Lin MH, Lee CP, Yang YH, Chen WC, Chang GH, Tsai YT, Chen PC, Tsai YH. Chang Gung Research Database: A multi-institutional database consisting of original medical records. Biomed J 2017;40:263-269.

6. Sim DS, Kim JH, Jeong MH. Differences in Clinical Outcomes Between Patients With ST-Elevation Versus Non-ST-Elevation Acute Myocardial Infarction in Korea. Korean Circ J 2009;39:297-303.

7. Zhang Q, Zhao D, Xie W, Xie X, Guo M, Wang M, Wang W, Liu W, Liu J. Recent Trends in Hospitalization for Acute Myocardial Infarction in Beijing: Increasing Overall Burden and a Transition From ST-Segment Elevation to Non-ST-Segment Elevation Myocardial Infarction in a Population-Based Study. Medicine (Baltimore) 2016;95:e2677.

8. Thiele H, Zeymer U, Neumann FJ, Ferenc M, Olbrich HG, Hausleiter J, de Waha A, Richardt G, Hennersdorf M, Empen K, Fuernau G, Desch S, Eitel I, Hambrecht R, Lauer B, Bohm M, Ebelt H, Schneider S, Werdan K, Schuler G, Intraaortic Balloon Pump in cardiogenic shock Ilti. Intra-aortic balloon counterpulsation in acute myocardial infarction complicated by cardiogenic shock (IABP-SHOCK II): final 12 month results of a randomised, open-label trial. Lancet 2013;382:1638-1645.

9. Mandawat A, Rao SV. Percutaneous Mechanical Circulatory Support Devices in Cardiogenic Shock. Circ Cardiovasc Interv 2017;10.

10. Brechot N, Hajage D, Kimmoun A, Demiselle J, Agerstrand C, Montero S, Schmidt M, Luyt CE, Lebreton G, Hekimian G, Flecher E, Zogheib E, Levy B, Slutsky AS, Brodie D, Asfar P, Combes A, International EN. Venoarterial extracorporeal membrane oxygenation to rescue sepsis-induced cardiogenic shock: a retrospective, multicentre, international cohort study. Lancet 2020;396:545-552.

11. Mehran R, Pocock SJ, Nikolsky E, Clayton T, Dangas GD, Kirtane AJ, Parise H, Fahy M, Manoukian SV, Feit F, Ohman ME, Witzenbichler B, Guagliumi G, Lansky AJ, Stone GW. A risk score to predict bleeding in patients with acute coronary syndromes. J Am Coll Cardiol 2010;55:2556-2566.

12. Dzavik V, Sleeper LA, Cocke TP, Moscucci M, Saucedo J, Hosat S, Jiang X, Slater J, LeJemtel T, Hochman JS, Investigators $S$. Early revascularization is associated with improved survival in elderly patients with acute myocardial infarction complicated by cardiogenic shock: a report from the SHOCK Trial Registry. Eur Heart J 2003;24:828-837. 
13. Hochman JS, Sleeper LA, Webb JG, Sanborn TA, White HD, Talley JD, Buller CE, Jacobs AK, Slater JN, Col J, McKinlay SM, LeJemtel TH. Early revascularization in acute myocardial infarction complicated by cardiogenic shock. SHOCK Investigators. Should We Emergently Revascularize Occluded Coronaries for Cardiogenic Shock. N Engl J Med 1999;341:625-634.

14. Damluji AA, Bandeen-Roche K, Berkower C, Boyd CM, Al-Damluji MS, Cohen MG, Forman DE, Chaudhary R, Gerstenblith G, Walston JD, Resar JR, Moscucci M. Percutaneous Coronary Intervention in Older Patients With ST-Segment Elevation Myocardial Infarction and Cardiogenic Shock. J Am Coll Cardiol 2019;73:1890-1900.

15. Fengler K, Fuernau G, Desch S, Eitel I, Neumann FJ, Olbrich HG, de Waha A, de Waha S, Richardt G, Hennersdorf M, Empen K, Hambrecht R, Fuhrmann J, Bohm M, Poess J, Strasser R, Schneider S, Schuler G, Werdan K, Zeymer U, Thiele H. Gender differences in patients with cardiogenic shock complicating myocardial infarction: a substudy of the IABP-SHOCK II-trial. Clin Res Cardiol 2015;104:71-78.

16. Koeth O, Zahn R, Heer T, Bauer T, Juenger C, Klein B, Gitt AK, Senges J, Zeymer U. Gender differences in patients with acute ST-elevation myocardial infarction complicated by cardiogenic shock. Clin Res Cardiol 2009;98:781-786.

17. Lee HJ, Lansky A, Mehta S, Haimi I, Salwan R, Zambahari R, Chen Y, Hassan F, Parise H, Fasila M, Kontsevaya A, Cheem TH, Baumbach A, Granger C, Grines C, Jacobs A, Tresukosol D, Soerianata S, Dy T. GENDER DISPARITIES IN ST-ELEVATION MYOCARDIAL INFARCTION CARE AND OUTCOMES IN EMERGING COUNTRIES: A GLOBAL LUMEN ORGANIZATION FOR WOMEN (GLOW) INITIATIVE AND CALL TO ACTION. J Am Coll Cardiol 2016;67:2356.

18. Zachura M, Wilczek K, Kurzawski J, Gierlotka M, Gasior M, Sadowski M. Gender-related differences in men and women with ST-segment elevation myocardial infarction and incomplete infarct-related artery flow restoration: a multicenter national registry. Postepy Kardiol Interwencyjnej 2018;14:356-362.

19. Cenko E, Yoon J, Kedev S, Stankovic G, Vasiljevic Z, Krljanac G, Kalpak O, Ricci B, Milicic D, Manfrini O, van der Schaar M, Badimon L, Bugiardini R. Sex Differences in Outcomes After STEMI: Effect Modification by Treatment Strategy and Age. JAMA Intern Med 2018;178:632-639. 


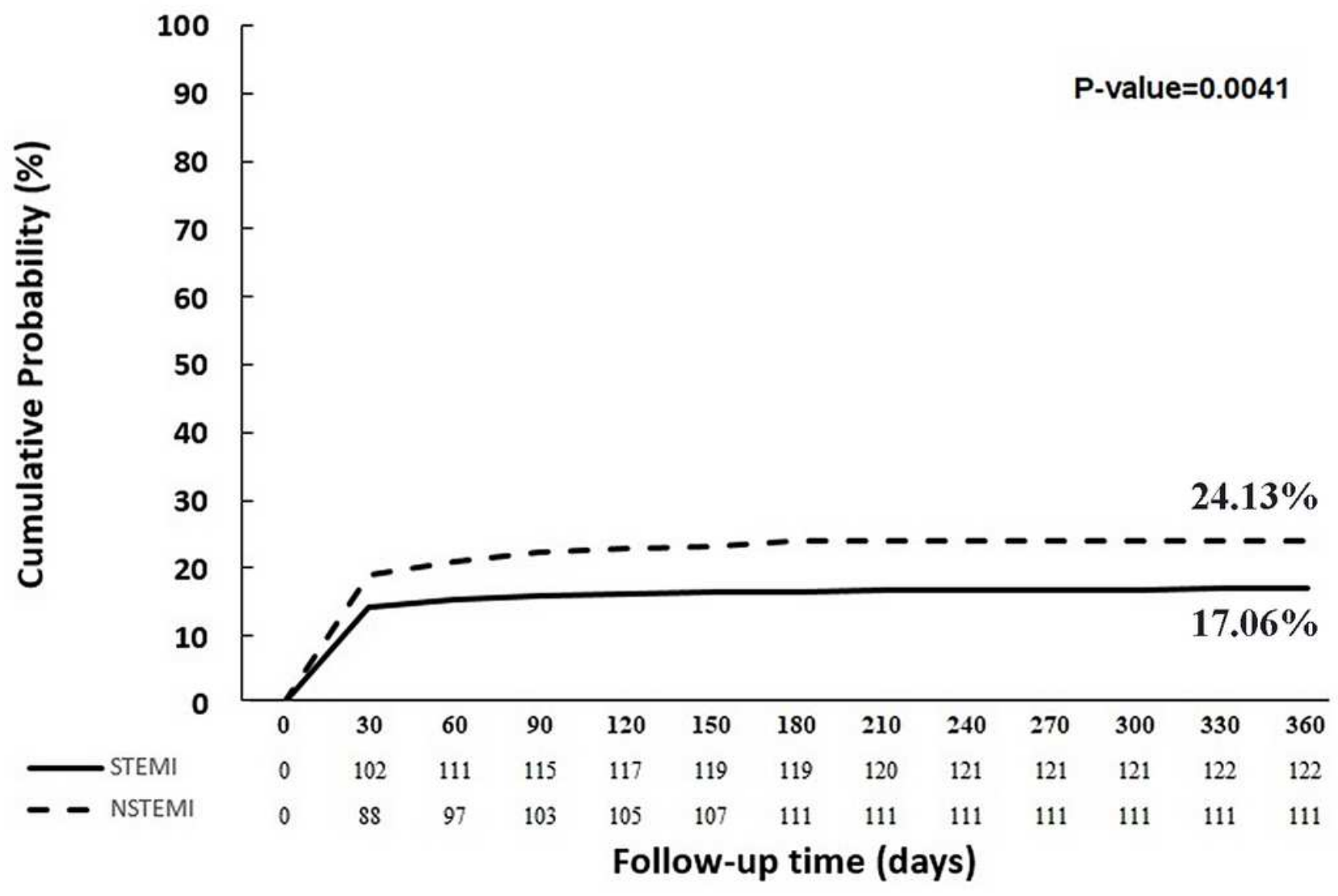

$-\operatorname{STEMI}(\%) \quad-\quad \operatorname{NSTEMI}(\%)$

\section{Figure 1}

1 year cumulative mortality of STEMI and NSTEMI patients complicated with cardiogenic shock
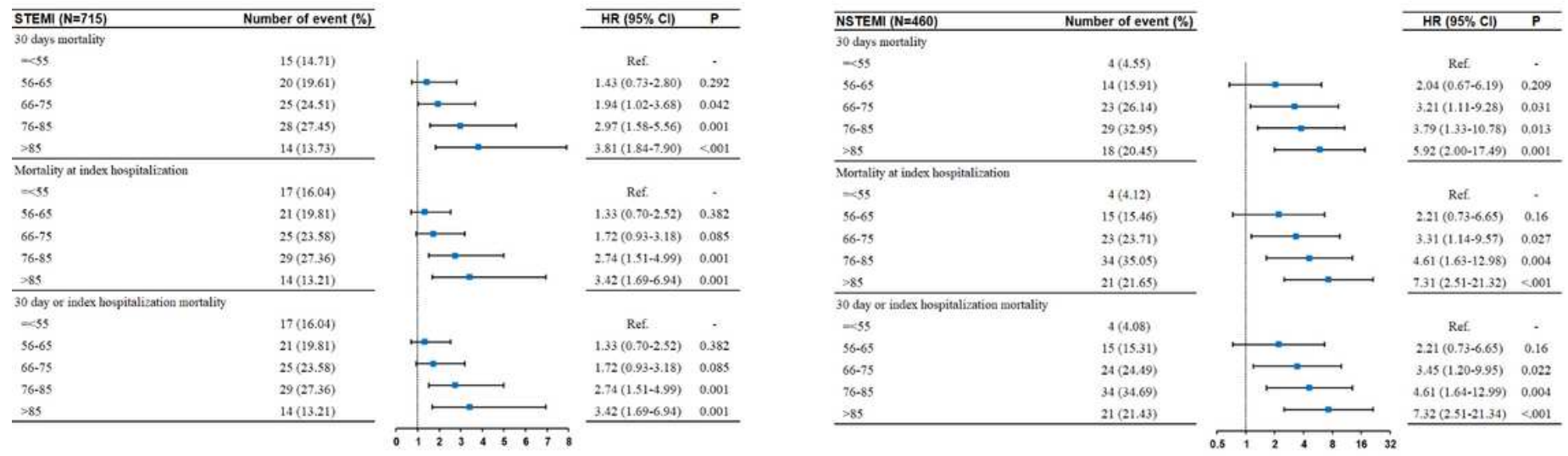

Figure 2

a. Subgroup analysis of short-term mortality for different age groups in STEMI. b. Subgroup analysis of short-term mortality for different age groups in NSTEMI. 


\section{Supplementary Files}

This is a list of supplementary files associated with this preprint. Click to download.

- Supplement20201027.docx 